\section{Endocarditis in Adult-onset Still Disease: A Case Report and Literature Review}

\section{To the Editor:}

Adult-onset Still disease (AOSD) is one of the most common autoinflammatory conditions responsible for fevers of unknown origin. AOSD is characterized by prolonged spiking fever, arthritis, evanescent rash, leukocytosis, and multiorgan involvement. Endocarditis, especially the right-sided type, is extremely rare in AOSD. Here we report a case of noninfective endocarditis in the tricuspid valve caused by AOSD.

An 18-year-old man who had a high fever and evanescent rashes in the chest for 3 weeks was admitted to our hospital. His fever was typically $>39^{\circ} \mathrm{C}$ and was accompanied by intermittent sore throat, polyarthralgia, and abdominal pain. There were 1 or 2 spiking fevers in a day (in the afternoon or evening), which showed good response only to dexamethasone. He had no history of other diseases and had not stayed in any epidemic areas. He stated that he did not have any drug addiction or allergies.

In physical examinations, he had tenderness in his shoulder and knee joints, no enlargement of lymph nodes, and had abdominal rigidity. Vital signs revealed fever and tachycardia. No throat swelling was found, and the patient had not undergone any special cardiopulmonary examinations.

Laboratory studies revealed hyperleukocytosis $\left(26.7 \times 10^{9} / 1\right.$ with $90.9 \%$ neutrophil), abnormal liver function (alanine transaminase $407 \mathrm{U} / 1$, aspartate transaminase $266 \mathrm{U} / 1$, gamma-glutamyl transferase $147 \mathrm{U} / 1$, alkaline phosphatase $150 \mathrm{U} / 1$ ), high acute-phase reactants (C-reactive protein 157.2 $\mathrm{mg} / \mathrm{l}$, erythrocyte sedimentation rate $85 \mathrm{~mm} / \mathrm{h}$, procalcitonin $4.5 \mathrm{ng} / \mathrm{ml}$ ), and extremely high serum ferritin $(>2000 \mu \mathrm{g} / \mathrm{l})$.

Serologic tests were negative for Epstein-Barr virus, cytomegalovirus, human immunodeficiency virus, syphilis, legionella, mycoplasma, Brucella, and hepatitis B and C viruses. Moreover, multiple bacterial cultures in blood, urine, and pharynx swab were all negative. Autoantibodies, such as antinuclear antibodies, antineutrophil cytoplasm antibodies, rheumatoid factor, anticardiolipin antibodies, and lupus anticoagulants were also negative. Iliac marrow biology revealed normal adult marrow, with negative culture results Positron emission tomography/computed tomography scanning revealed no mass and significantly deep lymphadenopathy. Echocardiography, however detected vegetation on the tricuspid valve (Figure 1 ). The size of vegetation was $5.9 \times 4.2 \mathrm{~mm}$ diameter, which caused slight tricuspid valve regurgitation.

According to the Modified Duke Criteria ${ }^{1}$, a working diagnosis of infective endocarditis was established. Correspondingly, ceftriaxone and linezolid were used for 12 days, but the spiking fever did not cease, and laboratory and echocardiography studies did not show any improvement. Given the failure of empirical treatment, the patient was suspected to have atypical cardiac manifestations of AOSD using Yamaguchi classification criteria $^{2}$. Antibiotics were stopped and prednisone $(2 \mathrm{mg} / \mathrm{kg}$ of body weight/d) was used. The patient's fever disappeared 2 days later and so did his accompanying symptoms. In the 3-month followup, the patient had no fever, and echocardiography revealed the vegetation on tricuspid valve had disappeared. We tapered the dosage of prednisone in the following 12 months, and he was symptom-free with normal laboratory tests.

It was challenging to diagnose AOSD, given its protean clinical manifestations and nonspecific abnormalities in laboratory tests. Initially, all treatments focused on the presumed culture-negative infective endocarditis (IE), yet some causes of culture-negative IE had been excluded by serology. Meanwhile, the clinical symptoms and examination abnormalities were not improved until the treatment with corticosteroid. Followup observation in the subsequent 12 months confirmed our diagnosis of AOSD. The possibility of cardiac valve involvement must be recognized in AOSD

We looked through related articles published before June 2017 from PubMed (MEDLINE) and Web of Science, with no restriction on study design or language. Only 7 cases of AOSD-caused endocarditis were reported $^{3,4,5,6,7,8,9}$, among which left-sided valves (including aortic and bicuspid valves) were commonly involved. Even so, it was challenging to confirm the existence of noninfective endocarditis in AOSD, especially the involvement of right-sided valves (mainly the tricuspid valve). To our
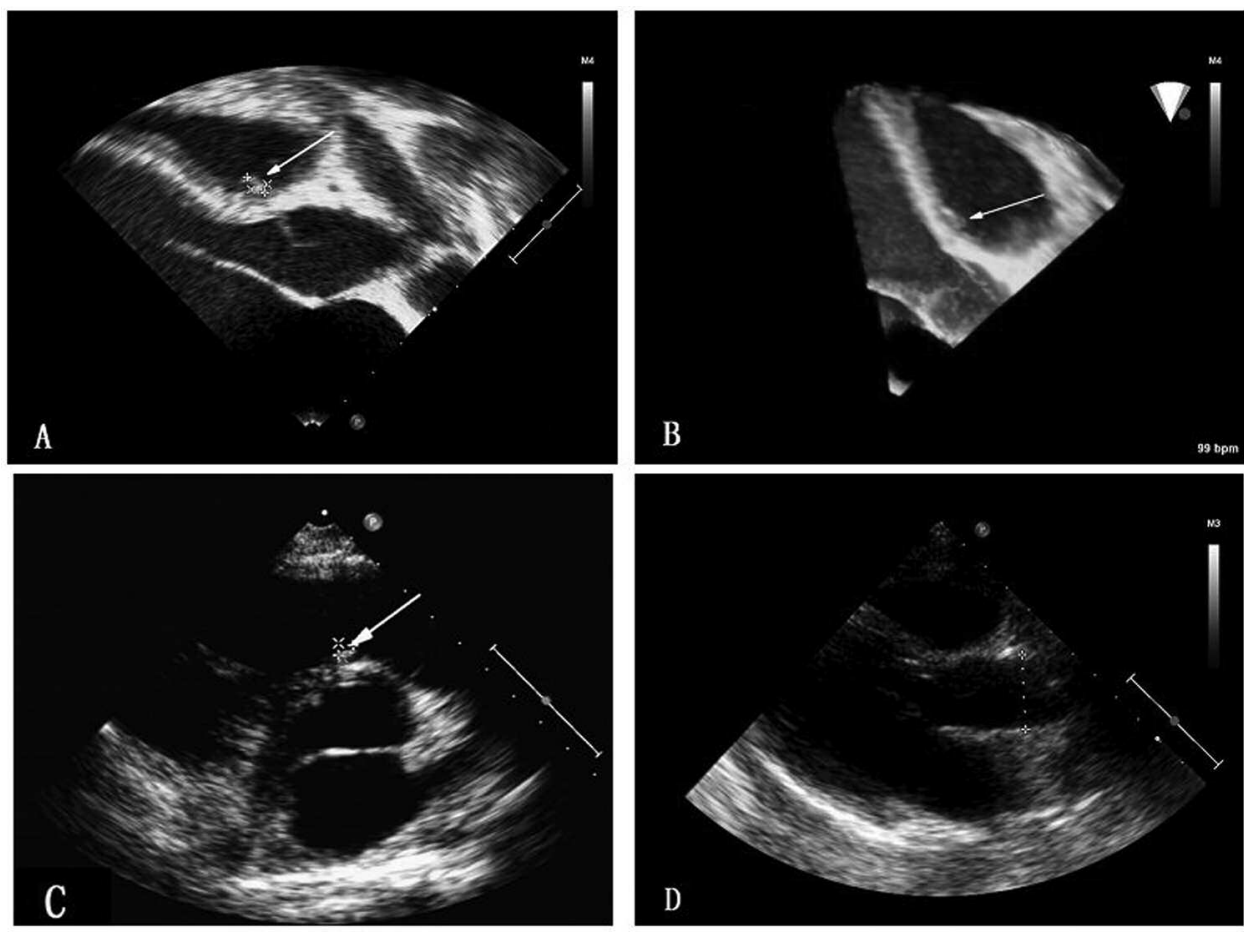

Figure 1. Findings on echocardiography. Transthoracic echocardiography (A) and transesophageal echocardiography $(B)$ detected vegetation on the tricuspid valve $(5.9 \times 4.2 \mathrm{~mm})$ upon patient admission. The size of the vegetation $(4.2 \times 2.6 \mathrm{~mm})$ was smaller 1 month after prednisone treatment $(\mathrm{C})$ and disappeared 3 months after prednisone treatment (D). 
knowledge, this is the second report about noninfective endocarditis in the tricuspid valve caused by AOSD.

In the literature, most patients had good responses to corticosteroids, and severe heart failure seldom happened, except in 1 female patient who had to undergo valve replacement ${ }^{9}$. Histological analysis of valve tissues revealed chronic active inflammation with neutrophilic infiltration. Avcin, et al presumed that vasculitis may be the basic change ${ }^{10}$. Therapeutic strategies of AOSD with endocarditis are similar to those of AOSD without endocarditis. Prednisone should be the first choice, yet there is no data concerning the effects of biological agents on endocarditis. Regression of AOSD with endocarditis may be much slower than without endocarditis ${ }^{2}$. Treatment for AOSD may be responsible for the regression of the valvular lesions observed in this condition.

Adult-onset Still disease can cause high-grade fever and noninfective endocarditis, which responds to prednisone.

\section{ACKNOWLEDGMENT}

The authors thank Dr. Bowen Zhao for providing the scanning by color Doppler echocardiography for our case.

XIN DANG, MD; YONGMEI HAN, MD; XINHUA ZHANG, MD, Department of Rheumatology, Sir Run Run Shaw Hospital, Zhejiang University, Hangzhou, China. Address correspondence to Dr. X. Zhang, Department of Rheumatology, Sir Run Run Shaw Hospital, Zhejiang University, No. 3 East QingChun Road, Hangzhou, China. E-mail: 3408234@zju.edu.cn. The present study was approved by the Ethics Committee of Zhejiang University, and written informed consent was obtained according to the Declaration of Helsinki.

\section{REFERENCES}

1. Li JS, Sexton DJ, Mick N, Nettles R, Fowler VJ, Ryan T, et al. Proposed modifications to the Duke criteria for the diagnosis of infective endocarditis. Clin Infect Dis 2000;30:633-38.
2. Yamaguchi M, Ohta A, Tsunematsu T, Kasukawa R, Mizushima Y, Kashiwagi H, et al. Preliminary criteria for classification of adult Still's disease. J Rheumatol 1992;19:424-30.

3. Ertugrul BM, Uyar G, Ozturk B, Sakarya S. A rare presentation of endocarditis in adult-onset Still's disease in diagnosis of fever of unknown origin. J Rheumatol 2012;39:198-9.

4. Garcia-Porrua C, Gonzalez-Juanatey C, Gonzalez-Gay MA Endocarditis in adult onset Still's disease: a 12 month followup. J Rheumatol 2001;28:2141-2.

5. Martin GA, Weir RA, Brown MC, Petrie CJ. Prosthetic valve, fever and arthropathy - still a difficult diagnosis. QJM 2014;107:213-5.

6. Taillan B, Fuzibet JG, Vinti H, Castela J, Ferrari E, Pesce A, et al. [Non-infectious mitral endocarditis during Still's disease in adults. A case with histologic study of the endocardium]. [Article in French] Ann Med Interne 1990;141:78-9.

7. Saraux A, Binard A, Devauchelle-Pensec V, Jobic Y, Jousse-Joulin S. Right-sided native valve endocarditis revealing adult-onset Still disease. Ann Intern Med 2008;149:517-9.

8. Zenagui D, De Coninck JP. Atypical presentation of adult Still's disease mimicking acute bacterial endocarditis. Eur Heart J 1995; 16:1448-50.

9. Lee JS, Do IN, Kang DH, Joo SJ, Yoo B, Moon HB, et al. Adult onset Still's disease as a cause of acute severe mitral and aortic regurgitation. Korean J Intern Med 2005;20:264-7.

10. Avcin T, Silverman ED, Forte V, Schneider R. Nasal septal perforation: a novel clinical manifestation of systemic juvenile idiopathic arthritis/adult onset Still's disease. J Rheumatol 2005;32:2429-31

J Rheumatol 2018;45:8; doi:10.3899/jrheum.171214 